\title{
Dll4-Notch signaling determines the formation of native arterial collateral networks and arterial function in mouse ischemia models
}

\author{
Brunella Cristofaro ${ }^{1, *}$, Yu Shi ${ }^{2,3, *}$, Marcella Faria ${ }^{1}$, Steven Suchting ${ }^{1}$, Aurelie S. Leroyer ${ }^{1}$, Alexandre Trindade ${ }^{4}$, \\ Antonio Duarte ${ }^{4}$, Ann C. Zovein ${ }^{5}$, M. Luisa Iruela-Arispe ${ }^{5}$, Lina R. Nih ${ }^{6}$, Nathalie Kubis ${ }^{6}$, Daniel Henrion ${ }^{7}$, \\ Laurent Loufrani ${ }^{7}$, Mihail Todiras ${ }^{2}$, Johanna Schleifenbaum ${ }^{7}$, Maik Gollasch ${ }^{7}$, Zhen W. Zhuang ${ }^{8}$, \\ Michael Simons ${ }^{8}$, Anne Eichmann ${ }^{1,8, \pm}$ and Ferdinand le Noble ${ }^{2,7,9, \mp}$
}

\begin{abstract}
SUMMARY
Arteriogenesis requires growth of pre-existing arteriolar collateral networks and determines clinical outcome in arterial occlusive diseases. Factors responsible for the development of arteriolar collateral networks are poorly understood. The Notch ligand Deltalike 4 (DII4) promotes arterial differentiation and restricts vessel branching. We hypothesized that DII4 may act as a genetic determinant of collateral arterial networks and functional recovery in stroke and hind limb ischemia models in mice. Genetic lossand gain-of-function approaches in mice showed that Dll4-Notch signaling restricts pial collateral artery formation by modulating arterial branching morphogenesis during embryogenesis. Adult $D / 14^{+/-}$mice showed increased pial collateral numbers, but stroke volume upon middle cerebral artery occlusion was not reduced compared with wild-type littermates. Likewise, D/l4 ${ }^{+/-}$mice showed reduced blood flow conductance after femoral artery occlusion, and, despite markedly increased angiogenesis, tissue ischemia was more severe. In peripheral arteries, loss of D/I4 adversely affected excitation-contraction coupling in arterial smooth muscle in response to vasopressor agents and arterial vessel wall adaption in response to increases in blood flow, collectively contributing to reduced flow reserve. We conclude that DII4-Notch signaling modulates native collateral formation by acting on vascular branching morphogenesis during embryogenesis. DII4 furthermore affects tissue perfusion by acting on arterial function and structure. Loss of D//4 stimulates collateral formation and angiogenesis, but in the context of ischemic diseases such beneficial effects are overruled by adverse functional changes, demonstrating that ischemic recovery is not solely determined by collateral number but rather by vessel functionality.
\end{abstract}

KEY WORDS: Angiogenesis, Arteriogenesis, Vessel branching, Dll4-Notch signaling, Mouse

\section{INTRODUCTION}

Arteries form highly branched networks that ramify throughout the body. Most arteries branch into progressively smaller diameter arterioles that in turn branch into even smaller sized capillaries, which supply oxygen and nutrients to tissues and organs. However, some arterioles directly connect with other arterioles, usually from a neighboring arterial tree, and form so-called native collateral arteries (Chalothorn and Faber, 2010). The native collateral circulation represents a major conduit for blood supply to tissues after occlusion of one of the feed arteries. Increases in shear stress and activation of inflammatory pathways upon occlusion trigger the

${ }^{1}$ CIRB Collège de France, Inserm U1050/CNRS UMR 7241, 75005 Paris, France. ${ }^{2}$ Max Delbrueck Center for Molecular Medicine (MDC), Robert Roessle Strasse 10, D13125 Berlin, Germany. ${ }^{3}$ Experimental and Clinical Research Center (ECRC) of the Charité and the MDC, D13125 Berlin, Germany. ${ }^{4}$ Centro Interdisciplinar de Investigação em Sanidade Animal (CIISA), Lisbon Technical University, 1300-477 Lisbon, Portugal. ${ }^{5}$ Department of Molecular, Cellular, and Developmental Biology, University of California Los Angeles, Los Angeles, CA 90095 USA. ${ }^{6}$ Inserm U965, Universite Paris Sorbonne, Paris Cite, 75475 Paris, France. ${ }^{7}$ Department of Neurovascular Biology, Inserm U1083, CNRS UMR6214, University of Angers, 49045 Angers, France. ${ }^{8}$ Yale Cardiovascular Research Center, Yale University School of Medicine, New Haven, CT 06520-8056, USA. ${ }^{9}$ Center for Stroke Research Berlin (CSB), Charité, D10117 Berlin, Germany.

*These authors contributed equally to this work

${ }^{\ddagger}$ Authors for correspondence (anne.eichmann@college-de-france.fr;

lenoble@mdc-berlin.de)

Accepted 4 February 2013 outward remodeling of these pre-existing bypasses (Arras et al., 1998; Eitenmüller et al., 2006). Their presence minimizes tissue injury after ischemia caused by atherosclerotic, atherothrombotic and thromboembolic vaso-occlusive disease, which are the major causes of morbidity and mortality in developed countries.

In addition to the extent of the native collateral circulation, tissue perfusion following an ischemic insult is enhanced by arteriogenesis, which can occur by enlargement of native collaterals or by de novo formation of arterioles, and by angiogenesis: the sprouting of new capillaries (Carmeliet, 2000). Numerous clinical attempts to increase angiogenesis in individuals with ischemic disease by administration of growth factors have failed (Grundmann et al., 2007), leading to the increased recognition that strategies to improve tissue perfusion need to focus on improving the extent of the collateral circulation and arteriogenesis (Schaper, 2009). Despite the clinical importance, factors influencing arterial branching morphogenesis and collateral network formation remain poorly characterized.

Here, we examined whether Delta-like 4 (D114)-Notch signaling, a pathway previously implicated in the regulation of arterial identity and angiogenic sprouting (Phng and Gerhardt, 2009; Swift and Weinstein, 2009) determines arteriogenesis and collateral vessel formation. D114 is a transmembrane ligand of Notch receptors that is selectively expressed in arterial endothelial cells and angiogenic tip cells during development (Benedito et al., 2009; Hellström et al., 2007; Shutter et al., 2000; Suchting et al., 2007). Dll4 ${ }^{-/}$mutant mice show reduced aorta size and ectopic expression of venous 
markers in the aorta, consistent with impaired arterial specification (Duarte et al., 2004; Gale et al., 2004). Conditional overexpression of Dll4 results in an enlarged aorta, and reduced vascular branching during embryogenesis (Trindade et al., 2008). Dll4 acts as a repressor of endothelial tip cell formation and loss of Dll4 stimulates excessive tip cell formation resulting in hyperbranching of retinal vessels (Hellström et al., 2007; Suchting et al., 2007). The Dll4Notch pathway regulates VEGF receptor expression in sprouting vessels and decreases VEGF responsiveness (Phng and Gerhardt, 2009). VEGF is a potent pro-angiogenic factor in ischemic conditions, and is thought to be a determinant of adult arteriogenesis in mice (Clayton et al., 2008). The cellular mechanism is believed to involve sprouting angiogenesis (Lucitti et al., 2012). Acute blockade of Notch signaling using an extracellular D114 decoy increases sprouting of ischemic capillaries following femoral artery ligation in mice (Al Haj Zen et al., 2010), indicating that D114-Notch signaling restricts post-ischemic angiogenesis. Likewise, Notch signaling blockade leads to hypersprouting of tumor vessels and decreases tumor growth because of failure to form a functional vascular network (Thurston et al., 2007).

In addition to negative effects on sprouting, Notch signaling has also been shown to have effects on vessel remodeling. Inhibition of Notch signaling prevented vessel regression in normal retinal development and in the oxygen-induced retinopathy model in mice (Lobov et al., 2011). D114/Notch inhibition increased the expression of vasodilators adrenomedullin and suppressed the expression of the vasoconstrictor angiotensinogen (a precursor of angiotensin II) in a VEGF-independent manner. Angiotensin II was shown to induce vasoconstriction and induced vessel regression, whereas angiotensin inhibitors inhibited vessel regression (Lobov et al., 2011). These observations suggested that Dll4-Notch inhibition might have beneficial effects on post-ischemic arteriogenesis by regulating vascular tone and preventing vessel regression. Finally, possible effects of Dll4-Notch signaling on formation of the collateral network have not been investigated yet.

Here, we have used genetic loss- and gain-of-function models in mice to define the role of Dll4 in arteriogenesis. We show that Dll4Notch signaling restricts collateral vessel formation by modulating arterial branching morphogenesis. We furthermore examined the relationship between collateral network size and functional recovery of ischemic tissues using stroke and hind limb ischemia models. Our results show that despite increased collateral vessel numbers, Dll4 ${ }^{+/-}$mice show poor blood flow recovery upon arterial occlusion. These results suggest that improved clinical and functional outcome after arterial occlusion do not simply rely on increasing vessel numbers, but rather on the quality of the recruited (neo) vessels.

\section{MATERIALS AND METHODS}

Mice

Dll4 $4^{+/}$heterozygous mice have been described previously (Duarte et al., 2004) and collateral vessel density was assessed by X-gal staining and after backcrossing onto Gja5 $5^{+/ \mathrm{eGFP}}$ mice (Miquerol et al., 2004). Both $\mathrm{Dll4} 4^{+/-}$ and $G j a 5^{+/ \mathrm{eGFP}}$ strains were maintained on a CD1 background. Cdh5Cre:Notch $1^{\text {flox/flox }}$ mice and conditional $m D l l 4$ gain-of-function mice have been described previously (Feil et al., 1996; Feil et al., 1997; Hellström et al., 2007; Deutsch et al., 2008; Trindade et al., 2008). Transgene expression induction was achieved by administration of doxycycline at $2 \mathrm{mg} / \mathrm{ml}$ in drinking water to the mothers from P0 up to P9. Mice were genotyped by PCR, assessing tail GFP and $\beta$-galactosidase staining. Whole-mount lacZ stainings were performed as described previously (Suchting et al., 2007). Animal experiments were carried out in accordance with European Community standards on the care and use of laboratory animals, with
German Law for the protection of animals and with National Institute of Health guidelines for care and use of laboratory animals; the protocols were approved by the local ethical committee.

\section{Inhibition of Notch activation}

To evaluate the role of Notch receptor signaling in collateral vessel formation, pregnant mice were injected intraperitoneally with DAPT $(100 \mathrm{mg} / \mathrm{kg}$ body weight) or vehicle (5\% ethanol/corn oil) at embryonic day E10.5, E11.5 and E12.5. Embryos were collected and analyzed at E13.5. Furthermore, newborn mice received either DAPT $(100 \mathrm{mg} / \mathrm{kg}$ body weight) or vehicle subcutaneously at postnatal day P2 and P4. Neonatal pups were culled at day P5 and P9, and pial collateral circulation was analyzed. Pregnant $C d h 5$-Cre:Notch $1^{\text {flox/flox }}$ female mice were injected with tamoxifen (1 mg/kg body weight) at E11.5 and embryos were analyzed at E13.5.

\section{Immunohistochemistry}

For whole-mount staining, dissected tissues/embryos were fixed in 4\% paraformaldehyde for 2 hours on ice and blocked overnight in blocking buffer $[\mathrm{PBS} / 0.5 \%$ blocking reagent (Perkin) $/ 0.3 \%$ Triton X-100/0.2\% BSA]. Tissues/embryos were incubated overnight at $4{ }^{\circ} \mathrm{C}$ with primary antibodies in blocking buffer (Cy3-conjugated anti-SMA, Sigma, 1:500) and anti-collagen IV (Novotec, 1:100). Tissues/embryos were washed in PBS $/ 0.3 \%$ TX-100 and incubated overnight with species-specific fluorescent secondary antibodies [Alexa 488 (Invitrogen), 1:200]. The samples were then washed in PBS/0.3\% TX-100 and mounted (Dako Fluorescent Mounting Medium, Dako). To determine vessel density on sections, muscles were excised, fixed with $4 \%$ phosphate-buffered paraformaldehyde, embedded in paraffin and sectioned at $4 \mu \mathrm{m}$. Capillaries were stained with isolectin B4 (1:25; Vector Labs) followed by Alexa Fluor 488 conjugated to streptavidin (1:100; Invitrogen); arteries in wild-type or $D l l 4^{+/}$mice backcrossed to arterial reporter $G j a 5^{+/ e G F P}$ were identified using an anti-GFP antibody (Abcam). Vessels were imaged using an Axioskope A1 microscope (Carl Zeiss MicroImaging, Jena, Germany) and a Coolsnap HQ fluorescent camera (Photometrics) connected to MetaMorph (Version 7.7.2.0) image analysis software.

\section{RNA and Taqman analysis}

Total RNA of femoral artery or gastrocnemius muscle was isolated using the RNeasy Mini Kit (QIAGEN). A total of $1 \mu \mathrm{g}$ RNA was used for cDNA synthesis (Thermoscript First-Strand Synthesis System; Invitrogen). Primers and probes were ordered from BioTez (Berlin, Germany). Real-time PCR amplification reaction was performed on a Sequence Detection System (7900 HT; Applied Biosystems). The comparative CT Method (DeltaDeltaCT Method) was used to analyze the data and GAPDH was used as an internal normalization control. The primer sequences and probes are listed in supplementary material Table S1.

\section{Middle cerebral artery occlusion}

The left middle cerebral artery of 8-week-old $\mathrm{Dll}^{+/-}$or wild-type mice was occluded as described previously (Nih et al., 2012). Forty-eight hours after the occlusion, mice were sacrificed and brains were collected. Coronal slices of forebrain were made using a vibratome (Leica) and were stained with 2\% 2,3,5-triphenyltetrazolium chloride (TTC, Sigma). Right and left hemispheres, and infarction volumes were measured using ImageJ software.

\section{Femoral artery occlusion model, assessment of blood flow with laser Doppler imaging technique}

Occlusion of the right femoral artery in 12-week-old mice was performed as described previously (Buschmann et al., 2010). For repetitive assessment of hindlimb blood flow after occlusion, we used the non-invasive laser Doppler imaging technique (LDF) (Buschmann et al., 2010). The LDF technique depends on the Doppler principle whereby low-power light from a monochromatic stable laser (wavelength $830 \mathrm{~nm}$, laser diode, model LDI2-HR, Moor Instruments, UK), incident on tissue is scattered by moving red blood cells, photo-detected and processed to provide a blood flow measurement. The ischemia score was determined as follows: 0 , normal; 1 , cyanosis or loss of nail(s); 2, partial or complete atrophy of digit(s); 3 , partial atrophy of forefoot. 

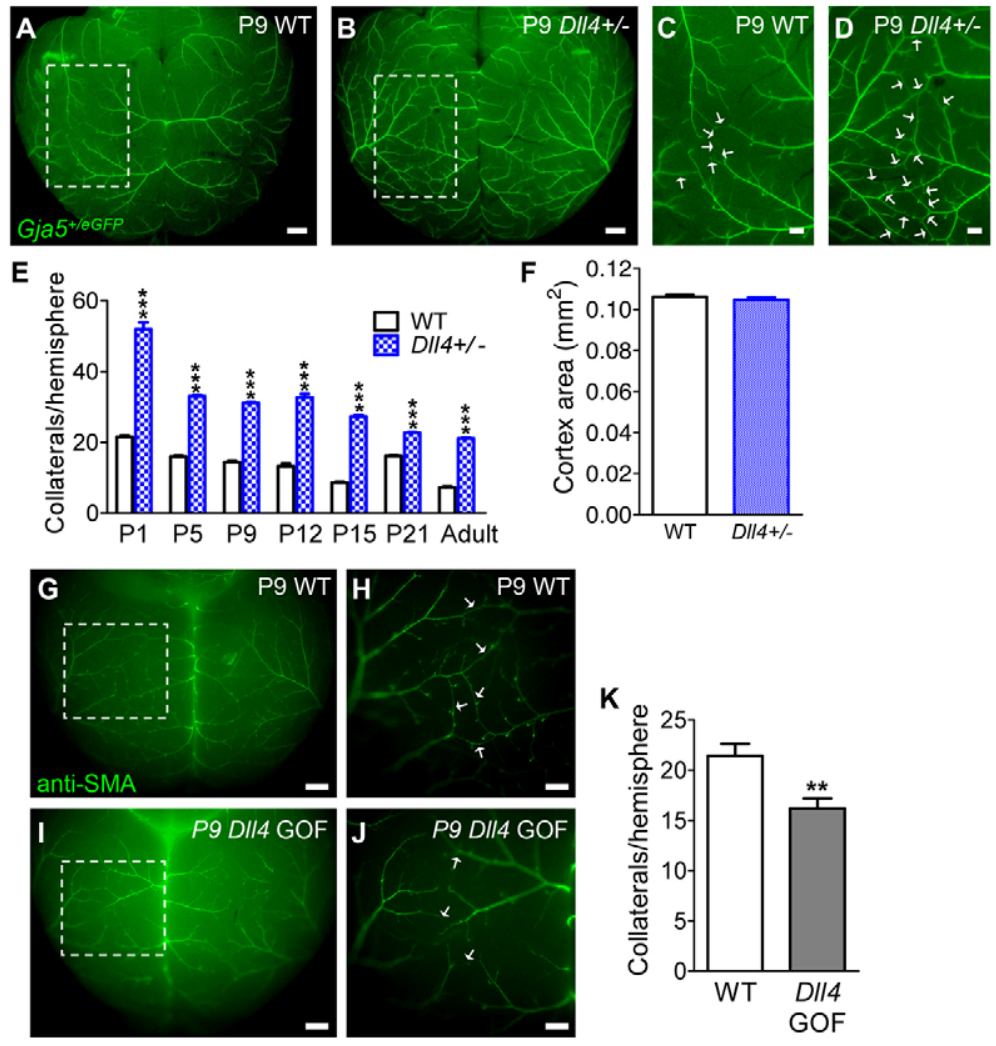

\section{Arterial blood pressure measurement}

Under isoflurane inhalation anesthesia, a catheter (Micro-Renethane $0.25 \times 0.12$ ) was inserted into the abdominal aorta through the left femoral artery for pressure (BP) measurements. After a 48-hour recovery, BP and heart rate (HR) were recorded with a transducer (MLT 1050 model) connected to a computer system for data acquisition and analysis (PowerLab, ADInstruments) in freely moving unanesthetized animals as described (Cayla et al., 2007).

\section{MicroCT imaging}

For visualization of the arterial collateral network after femoral artery occlusion, we performed $\mu \mathrm{CT}$ imaging (Zhuang et al., 2006). In short: 7 days after femoral artery occlusion, the abdominal aorta was cannulated and perfused with $100 \mathrm{mg} / \mathrm{kg}$ papaverin hydrochloride (Paveron, Weimer) to obtain maximal vasodilation, followed by perfusion with contrast agent. Hindlimb vascular network morphology was imaged and analyzed with a high resolution $\mu \mathrm{CT}$ imaging system, as described in detail previously (Zhuang et al., 2006).

\section{Measurement of flow induced remodeling and arteriolar contractility}

To study the effects of chronic changes in blood flow on arteriolar remodeling, we used the mesenteric ligation model as described previously (Buschmann et al., 2010; Loufrani et al., 2002). Arterioles of wild type and $D l l 4^{+/}$were exposed to high or low blood flow for a period of 7 days and isolated, and passive diameter-pressure curves were generated using the arteriograph. Pressure and diameter measurements were collected using a Biopac data acquisition system (Biopac MP100 and Acknowledge software; La Jolla, CA, USA).

To examine arteriolar contractility and relaxation behavior, resistance sized arterioles were isolated and mounted in a wire myograph as described previously (le Noble et al., 2000). To examine vasoconstriction, arterioles were challenged with norepinephrine (NE), phenylephrine (Phe) and angiotensin II (AngII). To examine vasorelaxation response, arterioles were pre-constricted with $35 \mathrm{mmol} / 1 \mathrm{~K}^{+}$and challenged with acetylcholine (ACh) or sodium nitroprusside (SNP). After examination of the arteries in the
Fig. 1. DII4 modulates pial arteriolar collateral number. (A-D) Representative microphotographs of collateral arteriolar connections (arrows in $C, D$ ) between the MCA and the ACA in

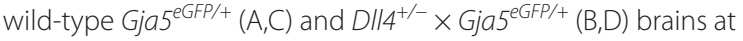
postnatal day $9(P 9)$. (C,D) Higher magnifications of boxes in A,B. There are more arterioles in DI/4+/- Scale bars: $500 \mu \mathrm{m}$. (E) Collateral arteriolar numbers per hemisphere at different time points after birth in wild-type and $D / 14^{+/-}$mice. Collateral density is highest at time of birth and decreases over time, indicating that pial collateral connections undergo remodeling in both wild type and $D / 14^{+/-}$. Data are shown as mean \pm s.e.m., $n=3-5$ mice at all time points. ${ }^{* *} P<0.001$ versus wild type. (F) Cortex area quantification at P24 shows no difference between wild type and $D / 14^{+/-}$. Values are mean \pm s.e.m., $n=5$ or 6 mice per group. (G-J) Representative microphotographs of collateral connections (arrows in $\mathrm{H}, J$ ) between MCA and ACA in wild-type $(\mathrm{G}, \mathrm{H})$ and D//4 gain-of-function (D//4 GOF; I,J) brains at

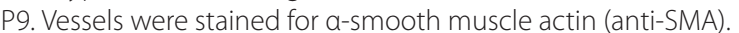
Scale bars $500 \mu \mathrm{m}$. (K) The number of collateral arterioles between MCA and ACA in wild-type and D//4 gain-of-function brains. Collateral numbers are decreased after DI/4 overexpression. Data are shown as mean \pm s.e.m., $n=13$ mice per group. ${ }^{* *} P<0.005$ versus wild type myograph, vessels were fixed with 4\% PFA under resting tension, isolated and embedded in paraffin wax. Media thickness was determined on $4 \mu \mathrm{m}$ sections stained with Lawson staining solution to visualize the lamina elastica interna and externa. Plasma leakage in hindlimb muscles was performed using Evans Blue as described previously (Di Lorenzo et al., 2009).

\section{Statistical analysis}

Data are expressed as the mean \pm s.e.m. $P$ values were calculated (SigmaStatPrism Software) using Student's $t$-test, Mann-Whitney U test or ANOVA, as indicated. $P<0.05$ was considered to be statistically significant.

\section{RESULTS}

\section{DII4 restricts pial collateral artery formation}

To examine whether Dll4 affects pial collateral growth, we used Dll4 ${ }^{+/}$-deficient CD1 mice carrying a beta-galactosidase reporter (Duarte et al., 2004) that were intercrossed with transgenic CD1 mice expressing EGFP driven by the arterial Gja5 (connexin 40) promoter $\left(\mathrm{Gja}^{+/ / \mathrm{GFP}}\right.$ mice) (Miquerol et al., 2004). X-gal staining and EGFP fluorescence allow detection of arteries and pial arteriolar-arteriolar collateral anastomoses between the middle cerebral artery (MCA) and anterior cerebral artery (ACA) (Fig. 1A-D) (Buschmann et al., 2010). In line with recent studies (Chalothorn and Faber, 2010), we find that wild-type mice show highest numbers of pial collaterals at P1, after which their numbers subsequently decrease to reach adult levels at P21 (Fig. 1A-E). At P1, Dll4 $4^{+-}$mice had about twofold more pial collateral connections compared with their wild-type littermates (Fig. 1A-E). Pruning of collaterals during early postnatal stages proceeded at the same rate in $\mathrm{Dll4^{+/ }}$ and in wild type, resulting in twice as many collaterals in adult $\mathrm{Dll}^{+/-}$mice (Fig. 1A-E). The changes in vascular density were independent of brain size as cortex area was comparable in wild-type and Dll4 heterozygous mice (Fig. 1F).

As a reduction in $D l l 4$ levels increased brain collateral numbers, we reasoned that conditional Dll4 overexpression should reduce 
native collateral formation. To test this, we used transgenic mice expressing Dll4 under the control of a tetracycline-inducible endothelial-specific Tie2 promoter (Trindade et al., 2008). Transgene expression was induced by administration of doxycyline in drinking water of the mother in the period between P0 and P9, and arteriolar collaterals were analyzed by immunostaining using an antibody directed against $\alpha$-smooth muscle actin ( $\alpha$ SMA) at P9 (Fig. 1G-J). Isolectin B4 immunostaining of retinal vessels of Dll4 gain-of-function mice showed a reduction in tip cell numbers, and a reduction in sprouting angiogenesis, indicating that induction of transgene expression was successful (supplementary material Fig. S1). Native collateral connections in brains of Dll4 gain-of-function mice were significantly reduced in comparison with controls $(P<0.01)$ (Fig. 1G-K). Taken together, these data show that native collateral formation in the brain is dependent on the dose of Dll4.

\section{DI/4 and Notch inhibition induce hyperbranching of embryonic MCA}

Evaluation of collateral vessel number showed significant differences between $D l l 4^{+/-}$and wild type already at the neonatal stage. Therefore, to establish when the number of collateral vessels starts to differ between wild type and $D l l 4^{+/}$, we extended our study to prenatal stages and analyzed branching morphogenesis of the MCA by whole-mount immunostaining using the vascular basement membrane marker collagen IV, of embryonic brains between E11.5 and E15.5 (Fig. 2). In wild-type mice, the MCA formed around E12.5 and was well developed by E13.5 and E14.5 (Fig. 2A-D,G; data not shown). MCA formation was slightly delayed in $\mathrm{Dll4^{+/- }}$ mice, and emerged as a clearly defined artery at E14.5. Once formed, the MCA of both $\mathrm{Dll4^{+/ }}$ and wild-type embryos developed one main branch, and a similar number of second-order arterioles branching off the main MCA trunk (Fig. 2A-D; supplementary material Fig. S2). The overall number of distal MCA artery branches was significantly increased in $D l l 4^{+/}$compared with wild-type littermates (Fig. 2G). As the distal branches of the MCA form pial collateral connections with the ACA (Chalothorn and Faber, 2010), these data suggest that increased collateral formation in $\mathrm{Dll}^{+/-}$mice is a direct consequence of MCA hyperbranching at the level of the distal parts of the MCA arteriolar tree.

To confirm that the effects of Dll4 on collateral formation are mediated via Notch receptors, we inhibited Notch signaling using the $\gamma$-secretase inhibitor DAPT. Administration of DAPT (intraperitoneally, $100 \mathrm{mg} / \mathrm{kg}$ body weight) into pregnant mice at embryonic days E10.5, E11.5 and E12.5 recapitulated distal MCA hyperbranching, as seen in $D l l 4^{+/}$embryos (Fig. 2E-G). We next genetically ablated Notch1 in endothelial cells using tamoxifeninducible Cdh5-Cre-ERT2:Notch $1^{\text {flox/flox }}$ transgenic mice. Cre activity was induced by injecting tamoxifen in pregnant mice at E11.5 (1 mg/kg body weight) and embryos were analyzed at E13.5. Despite low efficiency of Cre recombination (supplementary material Fig. S3A,B), distal MCA hyperbranching was evident in the $\mathrm{Cr}^{+}$group compared with $\mathrm{Cre}^{-}$controls (Fig. 2G; supplementary material Fig. S3A,B). We next explored the possibility that inhibition of Notch signaling during postnatal stages affects pial collateral numbers. Daily administration of DAPT during the first 4 postnatal days resulted in a sustained increase in collateral numbers at later stages (supplementary material Fig. S3C). Collectively, our data suggest that D114 signaling through Notch1 receptors restricts pial collateral formation by modulating branching morphogenesis of the distal region of the MCA and the ACA.
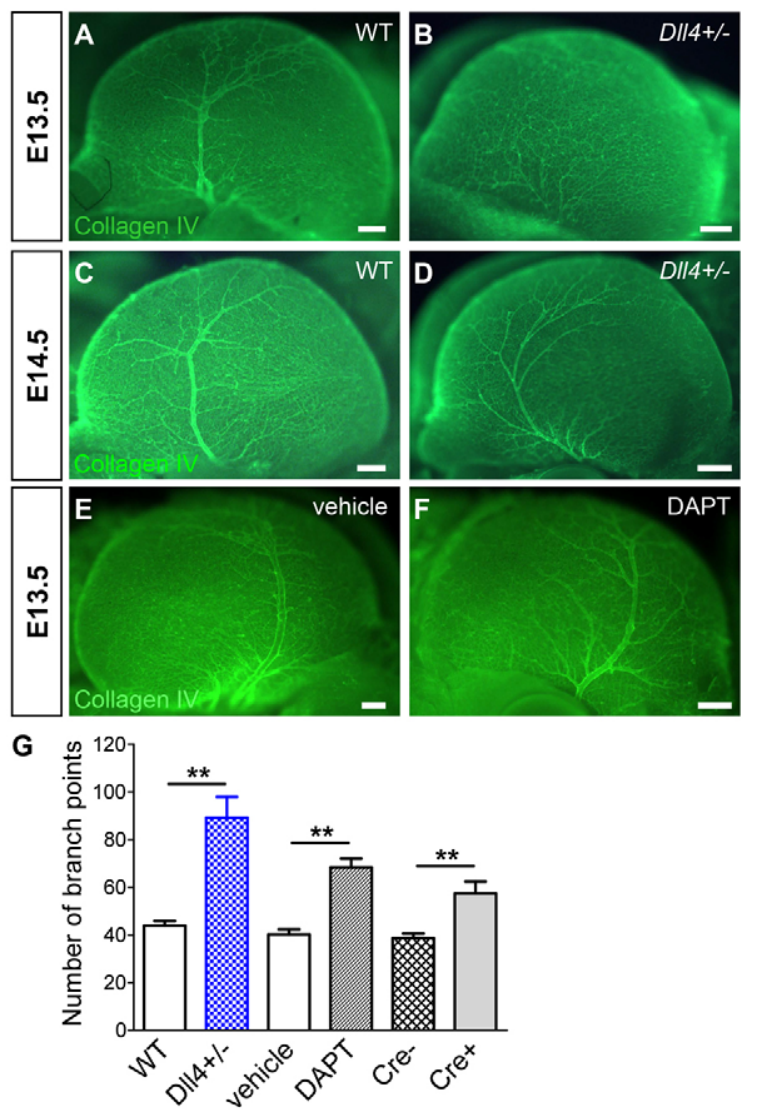

Fig. 2. Disruption of the DII4-Notch1 signaling pathway promotes embryonic MCA hyperbranching. (A-D) Epifluorescent images of wildtype and DI/4 ${ }^{+/-}$brains stained for collagen IV at embryonic stages E13.5 $(A, B)$ and E14.5 (C,D). (E,F) Epifluorescent images of collagen IV-stained brains at embryonic day E13.5 after treatment with DAPT (right panel) or vehicle (corn oil). MCA in DAPT mice shows increased branching similar to $D / 14^{+/-}$. Scale bars: $500 \mu \mathrm{m}$. (G) MCA branch point quantification. $\mathrm{Cre}^{-} / \mathrm{Cre}^{+}$: tamoxifen-inducible Cdh5-Cre-ERT2:Notch ${ }^{\text {flox/flox }}$ transgenic mice. Cre activity was induced by injecting tamoxifen in pregnant mice at E11.5 (1 mg/kg body weight) and embryos were analyzed at E13.5. ${ }^{*} P<0.01 . n=5$ mice per group. Values are mean \pm s.e.m.

\section{Increased collateral density in $\mathrm{D} / 14^{+/-}$mice or in mice treated embryonically with DAPT does not ameliorate post-ischemic recovery after MCA occlusion}

As stroke volume upon MCA occlusion has been shown to correlate with pial collateral density in mice (Zhang et al., 2010), we reasoned that heterozygous $\mathrm{Dll} 4^{+/-}$animals might be protected from strokeinduced injury. To test this hypothesis directly, we performed MCA occlusion and measured stroke volume in $\mathrm{Dll} 4^{+/-}$and wild-type littermates (Fig. 3A,B). Despite increased collateral density in the pial circulation, stroke volume upon MCA occlusion was not significantly different between $\mathrm{Dll} 4^{+/-}$and wild type at day 2 postocclusion (Fig. 3C). Moreover, $\mathrm{Dll}^{+/-}$brains frequently showed hemorrhagic lesions (Fig. 3B, blue arrow), which were not observed in wild-type controls. This suggests additional functional defects in Dll4 ${ }^{+/-}$pial collaterals.

We next tested whether transient loss of Notch signaling during embryonic development can augment collateral number and decrease stroke volume. To achieve this, we treated pregnant mice 

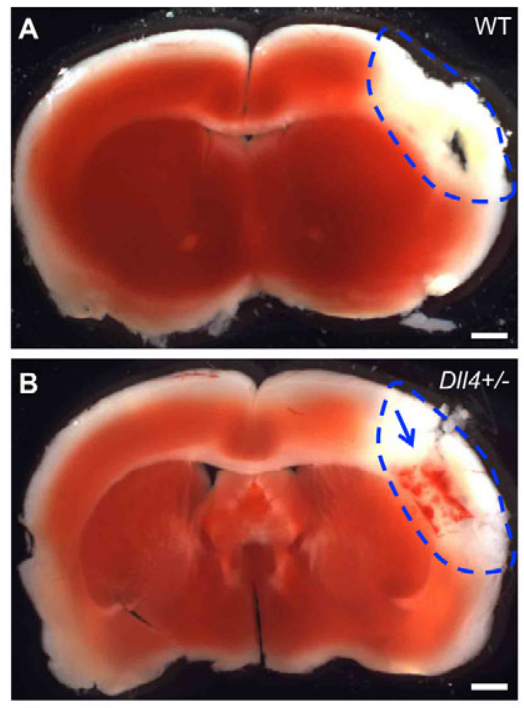

C

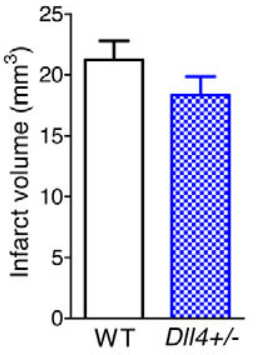

Fig. 3. Stroke volume after middle cerebral artery occlusion.

$(\mathbf{A}, \mathbf{B})$ Representative images of TTC-stained brain slices of wild-type and DII4+- mice 2 days after MCA occlusion. The infarcted area is demarcated by the broken blue line. Hemorrhagic areas are present in the infarct zone in DI/4 ${ }^{+-}$(B, blue arrow), which are not observed in wild type. Scale bars: $1 \mathrm{~mm}$. (C) Quantification of stroke volume in wild-type and D//4 ${ }^{+-}$brains at day 2 post-MCAO. Stroke volume is comparable between $\mathrm{D} / 4^{+/-}$and wild type.

with DAPT at E10.5, E11.5 and E12.5, and let the mice give birth and grow pups to adulthood. DAPT-treated mice showed an increased number of collateral vessels compared with vehicletreated (corn-oil injected) mice, indicating that it is possible to increase adult collateral vessel number by transiently interfering with Notch signaling during embryonic development (supplementary material Fig. S4A). Mice were then subjected to middle cerebral artery occlusion. We find that stroke volume was not significantly different between DAPT-treated and control animals (supplementary material Fig. S4B). Therefore, both $\mathrm{Dll4}{ }^{+/-}$ mice and mice with transient Notch inhibition induced by treatment with DAPT during embryogenesis showed increased pial collateral numbers, but no changes in stroke volume upon MCA occlusion.

\section{DII4 ${ }^{+-}$mice show reduced blood flow recovery upon femoral artery occlusion}

To further characterize the role of Dll4 in adaptive vascular remodeling upon arterial occlusion, we used the mouse hindlimb ischemia model and examined angiogenesis, arteriogenesis and functional recovery after occlusion of the femoral artery (FAO). Functional blood flow recovery was assessed with laser Doppler flow (LDF) imaging (Fig. 4A,B). Prior to FAO, hindlimb perfusion was similar between $\mathrm{Dll}^{+/-}$and wild-type littermates (Fig. 4A,B).
FAO reduced hindlimb perfusion in $\mathrm{Dll}^{+/-}$and wild-type mice to a similar extent, and perfusion remained comparable until day 1 post-FAO (Fig. 4B). However, between day 3 and day 21 postocclusion, perfusion recovery was significantly lower $(P<0.001)$ in $D l l 4^{+/}$compared with wild type (Fig. 4A,B). As a result, the ischemia score was significantly higher in $\mathrm{Dll4}^{+/-}$(Fig. 4C). Angiogenic responses in $\mathrm{Dll4} 4^{+/}$hindlimb were more pronounced compared with wild type, as highlighted by increased capillary density and capillary to fiber ratios in the ischemic muscle of Dll4 $4^{+/}$hindlimb (Fig. 4D,E), consistent with previous studies using acute Notch signaling blockade (Al Haj Zen et al., 2010). Using Evans blue to detect plasma leakage, we observed increased plasma leakage in $\mathrm{Dll}^{+/-}$, especially in the gastrocnemius muscle (Fig. 4F,G), indicating that the increased angiogenesis in the ischemic hindlimb of $\mathrm{Dll}^{+/-}$mice generated immature leaky vessels.

MicroCT imaging and histological analysis in $\mathrm{Dll}^{+/-}$and wildtype unligated control hindlimbs showed comparable arteriolar densities in both $\mathrm{Dll4}^{+/-}$and wild type (supplementary material Fig. S5A-C). However, in the occluded ischemic hindlimb we detected significantly lower arteriolar numbers in $\mathrm{Dll4^{+/- }}$ mice when compared with wild-type littermates (Fig. 5A-C), especially in $\mathrm{Dll}^{+/-}$calf muscle (Fig. 5C,D), indicating that, in addition to defective angiogenesis, arteriogenesis is impaired in $\mathrm{Dll4} 4^{+/-}$mice. To rule out that reduced flow recovery was due to altered input arterial pressure, we measured heart rate and blood pressure in $D l l 4^{+/-}$and wild-type littermates. Baseline mean arterial blood pressure (MAP) was slightly elevated in $\mathrm{Dll4}^{+/}$(supplementary material Fig. S6A) and heart rates (HR) were comparable with wild type (supplementary material Fig. S6B). Thus, it is unlikely that a reduction in arterial pressure accounted for the perfusion deficit in Dll4 ${ }^{+/}$mice.

\section{$D / 14^{+/-}$mice show no defect in arterial specification}

Formation of functional perfused arterial networks requires specification of arterial identity in vessels (Swift and Weinstein, 2009). To examine possible arterial specification defects, we analyzed regulators of arterial identity in the ischemic hindlimb. Expression of Dll4 was, as expected, significantly lower in both the femoral artery (FA) $(P<0.05)$ and the gastrocnemius muscle (Gastro) $(P<0.01)$ of $\mathrm{Dll}^{+/-}$mice (supplementary material Fig. S7A,B). Both control and ischemic hindlimb expressed Dll4 in blood vessels (supplementary material Fig. S7C). To test whether Dll4 haplo-insufficiency impaired the ability to activate the pathways essential for arterial differentiation, we measured expression of Hifla, Hiflb (Arnt - Mouse Genome Informatics), Vegfa, Kdr, Notch1 (Fig. 6A-E), and the Notch downstream effector ephrin B2 (Efnb2) at day 7 post-FAO (Fig. 6F). Expression of Efnb2 (Fig. 6F) was significantly upregulated upon FAO in $\mathrm{Dll}^{+/-}$, and significantly reduced expression of the other genes was never observed, thus suggesting that the arterial specification pathway is functional. As specification signaling appears normal, we next considered whether changes in arteriolar function might underlie the observed reduction in perfusion.

\section{DII4 ${ }^{+/-}$arterioles show altered functional responses to vasopressor agents and flow}

To test for functional changes, we challenged resistance arterioles from $D l l 4^{+/-}$and wild-type mice with vasoconstrictors and dilators, and measured force development using the myograph (Fig. 7). The maximal contractile responses to norepinephrine (NE), the $\alpha 1$ - 

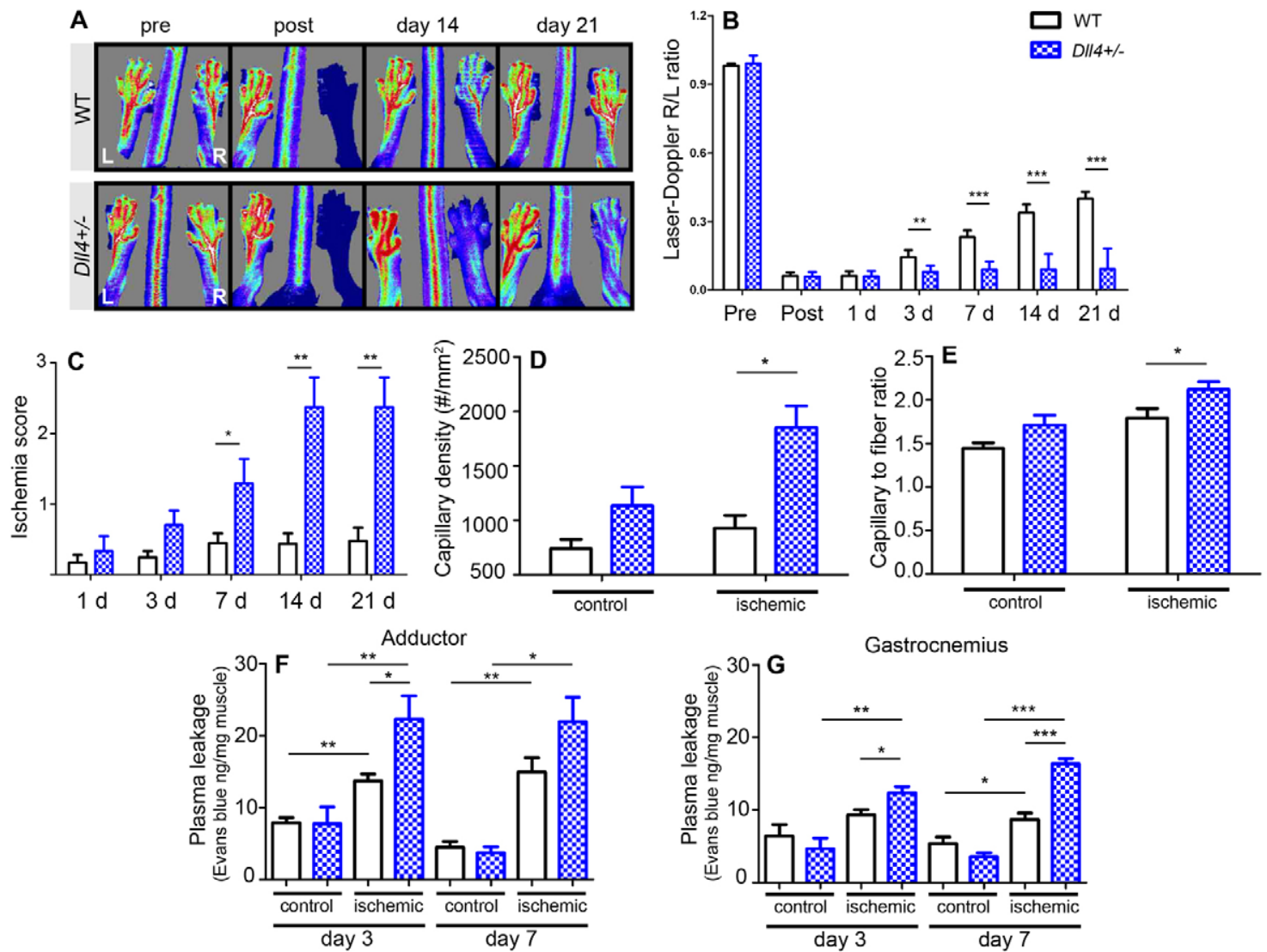

Fig. 4. DII4 ${ }^{+/-}$mice show reduced functional recovery after femoral artery occlusion. (A) Representative laser Doppler flow (LDF) images before and at various time points after femoral artery occlusion (FAO). (B) LDF perfusion shown as ratio of right to left hindlimb (R/L ratio) in wild type ( $n=20$ ) and $D / / 4^{+/-}(n=6)$. There is reduced perfusion recovery in $D / / 4^{+/-}$. (C) Ischemia score after FAO shows increased ischemia in D//4 ${ }^{+/-}$. (D,E) Quantitative analyses of capillary density (D) and capillary-to-fiber ratio (E) on sections of the gastrocnemius muscle. (F,G) Assessment of vascular leakage using Evans blue dye in wild-type and D//4+/- adductor muscle (F) and gastrocnemius muscle (G) at 7 days after FAO. Pre, pre-ligation; post, immediately after ligation. L, left hindlimb; $R$, right hindlimb. (F,G) Day 3: wild type, $n=6 ; D / / 4^{+/-}, n=6$. Day 7: wild type, $n=8 ; D / / 4^{+/-}, n=4 .{ }^{*} P<0.05,{ }^{* *} P<0.01,{ }^{* * *} P<0.001$.

adrenergic agonist phenylephrine (Phe) and angiotensin II (Ang II) were significantly larger in $\mathrm{Dll4^{+/ }}$ (Fig. 7A,C,D). Importantly, the maximal contractile response to receptor independent depolarization using $125 \mathrm{mmol} / 1 \mathrm{~K}^{+}$-Krebs was also significantly larger in Dll4 ${ }^{+/-}$ (Fig. 7A). As arteriolar media thickness (reflecting muscle mass), was comparable between wild type and $\mathrm{Dll4}^{+/-}$(Fig. 7B), increased contractile responses to vasopressor stimuli most likely resulted from changes in smooth muscle excitation-contraction coupling.

The nitric oxide (NO) pathway is activated during the arteriogenesis process and NO acts as a major vasodilator in arterioles, enforcing blood flow delivery. Arteriolar relaxation in response to the NO-dependent vasodilator acetylcholine (Fig. 7E), as well as the effect of the NO-synthase inhibitor L-NAME (Fig. 7F), were comparable between $\mathrm{Dll}^{+/-}$and wild type. In addition, arteriolar relaxation responses to the NO donor sodium nitroprusside (SNP) were similar (Fig. 7G). Thus, the NOdependent vasorelaxation pathway is functioning adequately in Dll4 ${ }^{+/}$mice.

The flow-induced outward remodeling response is essential for growth and expansion of collateral networks (Eitenmüller et al., 2006). We exposed arterioles of wild-type and $\mathrm{Dll4}^{+/}$mice to a high or low flow regime, for a period of 1 week, and measured the structural lumen adaptation using the arteriograph (Fig. 8A). We observed that the flow-induced outward remodeling response was severely blunted in $\mathrm{Dll4}^{+/}$when compared with wild type (Fig. 8BD). In wild-type arterioles exposure to a high flow increased lumen size by about $30 \%$, whereas in $\mathrm{Dll4}^{+/-}$this response was less than $5 \%(P<0.05)$ (Fig. 8D). Low flow-induced inward remodeling responses were comparable in both groups (Fig. 8B-D). Taken together, $\mathrm{Dll} 4^{+/-}$arterioles, when challenged by increased blood flow, will hardly enlarge, thus predicting impaired expansion of collateral networks upon FAO. Collectively, $\mathrm{Dll}^{+/-}$arterioles show impaired responses to shear stress and to vasoactive molecules that negatively affect blood flow conductance; this lack of response renders the arteriolar network susceptible to rarefaction.

\section{DISCUSSION}

Formation of extensive collateral networks plays a life-saving role in ischemic cardiovascular diseases (Meier et al., 2007). Although stimulation of collateral growth and arteriogenesis has emerged as one of the principle revascularization approaches, factors responsible for the presence and expansion of collateral networks are poorly understood. A recent report (Lucitti et al., 2012) indicates that VEGF-mediated sprouting events during crucial stages of embryo development can contribute to collateral formation in the pial circulation. VEGF acts upstream of D1l4-Notch signaling, and here we show that D114-Notch signaling acts a genetic determinant of native arterial collateral number. In the mouse brain, native 
A

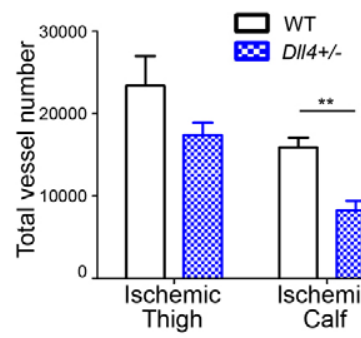

B
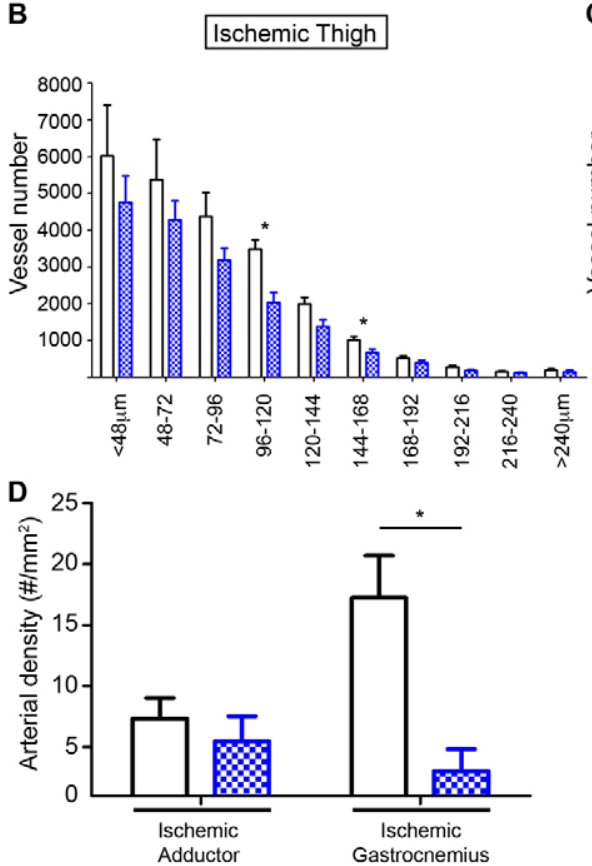

Fig. 5. DII4 ${ }^{+/-}$mice show reduced arteriolar numbers in the ischemic hindlimb. (A) $\mu \mathrm{CT}$ reconstruction and quantification of arteriolar number at $16 \mu \mathrm{m}$ resolution of thigh and calf portions of wild type and $\mathrm{D} / 4^{+/-}$at 7 days after FAO. (B,C) Quantitative analysis of $\mu C T$ images in the ischemic thigh (B) and the ischemic calf (C) as total number of vascular structures per indicated diameter class in consecutive $z$-axis slices. (D) Histological analysis of arteriolar density in wild-type $(n=7)$ and DI/4+- $(n=3)$ adductor and gastrocnemius muscle 7 days after FAO. (A-C) Wild type, $n=4$; $D / 14^{+/-}, n=4$; ${ }^{*} P<0.05$, ${ }^{* *} P<0.01$. arteriolar collateral networks appear as distal end-to-end arteriolar anastomoses and are detectable at time of birth (Chalothorn and Faber, 2010). We show that the extent of such native collateral networks inversely relates to $D l l 4$ gene dosage, with a high number of collaterals in Dll4 loss-of-function mice, and low collateral number in Dll4 gain-of-function mice. The effect of Dll4 on native collateral formation is Notch dependent, as both inhibition of Notch signaling with DAPT and endothelial-specific deletion of Notch1 phenocopy the Dll4 loss-of-function phenotype. We show that, in the brain, increased collateral number in $\mathrm{Dll4}{ }^{+/-}$mice results from hyperbranching of the most distal parts of the embryonic middle cerebral arteriolar tree, leading to increased end-to-end anastomosis formation with likewise increased ACA branches. Although positioning of the main MCA trunk and formation of second order side branches is not altered in Dll4- or Notch-deficient mice, arterial formation is slightly delayed; from E13.5 onwards, Dll4 ${ }^{+/}$and Notch-deficient mice display a significant increase in distal MCA arteriolar branches when compared with age-matched wild-type littermates. Thus, loss of Notch-D114 signaling enhances distal arterial branching morphogenesis, culminating in more native collaterals in the neonatal pial circulation.

To our surprise, functional recovery and ischemic outcome in stroke and hindlimb ischemia models were not improved in $\mathrm{Dll}^{+/-}$ mice, despite the clear increase in collateral vessel number. We also find that transient Notch inhibition using short-term DAPT treatment during embryonic development can increase collateral vessel number, but also in this setting no functional improvement on post-ischemic recovery is seen in adult mice. The reason for this defect is likely to be the defective vessel function. However, it might be possible to find a developmental time window during which DAPT treatment will improve collateral vessel number without adversely affecting vessel function and remodeling. Our data show that postnatal treatment with DAPT also leads to increased collateral vessel number; therefore, it might be possible that DAPT treatment during this time window will have positive effects on post-ischemic recovery.

These results show that the impact of collateral networks on functional flow recovery is not solely determined by augmenting vessel numbers, but rather by vessel functionality. We find increased vascular proliferation and capillary network formation associated with plasma leakage in $\mathrm{Dll} 4^{+/-}$mice after femoral artery occlusion, in line with previous reports describing non-productive angiogenesis in mice after inhibition of Notch using Ad-driven soluble D114, or treatment with D114 neutralizing antibodies in the setting of tumor angiogenesis (Noguera-Troise et al., 2006; Scehnet et al., 2007; Thurston et al., 2007). Reduced post-ischemic flow recovery in $\mathrm{Dll} 4^{+/-}$mice may therefore be a direct consequence of the immature dysfunctional capillary bed. However, these effects occur downstream of the collateral arterial network, which is increased, not decreased in $\mathrm{Dll} 4^{+/-}$mice, and must therefore be due to alterations in arteriolar architecture and function upstream of the capillary network.

As Dll4 is required for arterial differentiation, we determined whether loss of a single $\mathrm{Dll} 4$ allele impedes arterial specification in the hindlimb vasculature. Expression analysis indicates that this is not the case, and the Notch downstream effector - arterial marker 

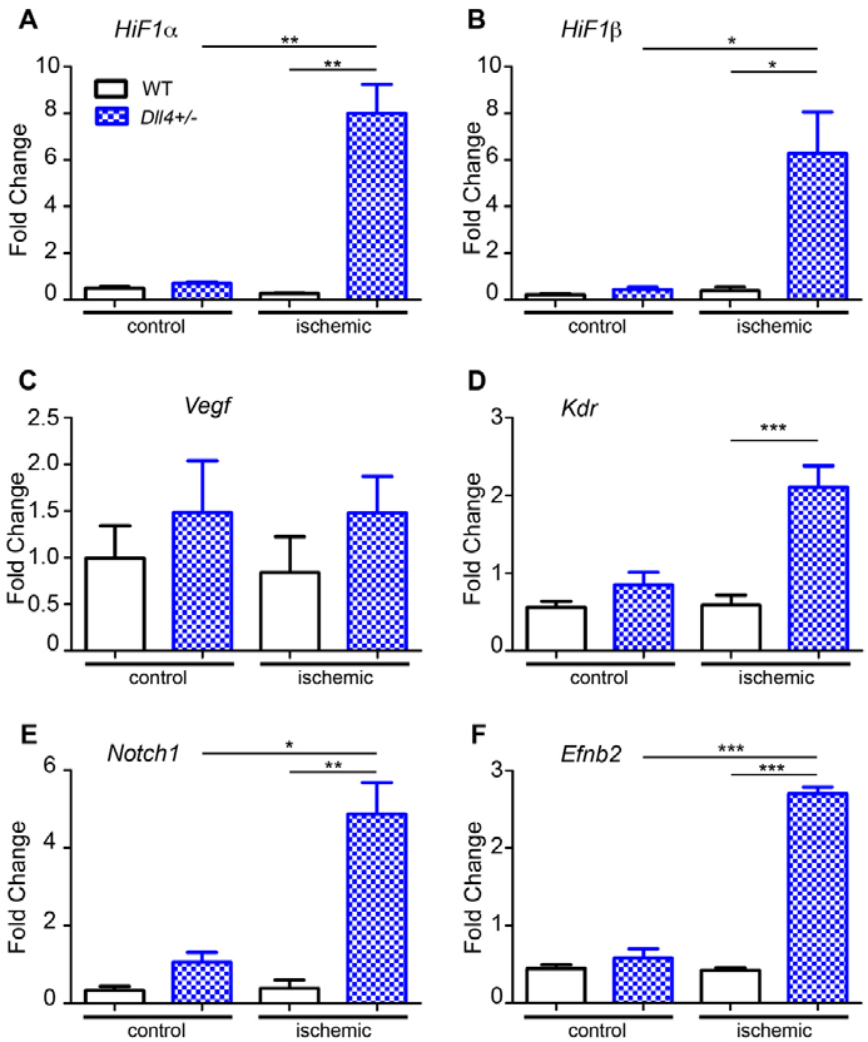

Fig. 6. Arterial specification genes are not suppressed in $\mathrm{Dll} 4^{+/-}$ mice. (A-F) Quantitative real-time PCR analysis of indicated genes in gastrocnemius muscle at 7 days after FAO. (A) Expression of hypoxia inducible factor 1a (Hif1a), (B) hypoxia inducible factor $1 \beta$ (Hif1b), (C) vascular endothelial growth factor A (Vegfa), (D) Kdr, (E) Notch1 and (F) ephrin B2 (Efnb2). $n=5$ or 6 mice per genotype and data point. Data are shown as mean \pm s.e.m. ${ }^{*} P<0.05,{ }^{* *} P<0.01,{ }^{* *} P<0.001$.

Efnb2, is upregulated in Dll4 ${ }^{+/}$ischemic hindlimb. Recruitment of collateral networks is triggered by hemodynamic factors, most notably increases in shear stress (Eitenmüller et al., 2006). Dll4 ${ }^{+/}$ arterioles show striking abnormalities in vascular remodeling responses to increases in shear stress; structural outward remodeling responses in wild type are about sixfold higher than in $\mathrm{Dll}^{+/-}$. This means that $D l l 4^{+/-}$arterioles, when exposed to high flow, almost fail to make a structurally larger lumen. Following Poiseuille's law, reduced diameter growth predicts diminished perfusion recovery, which is indeed in line with the $D l l 4^{+/-}$hindlimb LDF measurements. Furthermore, a failure to induce a rapid increase in collateral diameter causes a sustained hypoperfusion of the downstream areas, and here lack of sufficient blood flow and shear stress signal is by itself a trigger for vessel pruning. In the mouse retina IOR model, Dll4-Notch affects blood flow perfusion and microvessel maintenance by regulating expression of vasoactive molecules (Lobov et al., 2011). In addition, we find that Dll4 affects excitation contraction coupling in arterioles, relevant for controlling vascular tone. The concept that emerges from these observations is that in developing arterioles Dll4 acts on arterial specification signaling, whereas in more mature arteries it modulates arteriolar function. Such a bimodal effect on structure and function may further fine-tine tissue perfusion and oxygen homeostasis.

Factors regulating arteriolar function downstream of Dll4-Notch signaling remain to be identified. Previous work has shown that
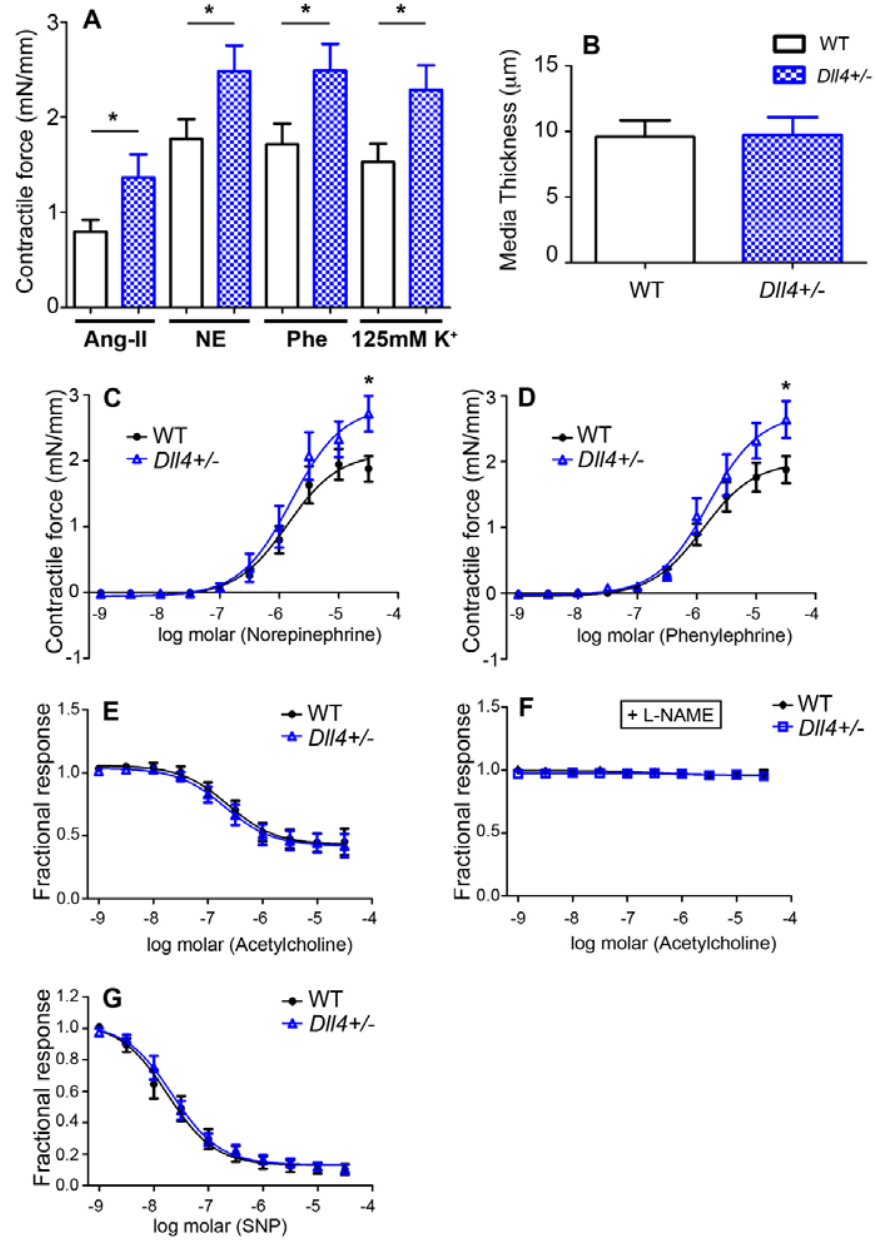

Fig. 7. DII4 ${ }^{+/-}$mice show increased arteriolar contractility.

(A) Contractile force per unit vessel length of mesenteric resistance arteries examined in a myograph: after challenge with angiotensin II (Ang II), norepinephrine (NE), phenylephrine (Phe) or direct depolarization using $125 \mathrm{mmol} / \mathrm{I} \mathrm{K}$ Krebs buffer. Contractility is increased in D//4 ${ }^{+/-}$.

(B) Vessel wall media thickness was comparable between wild type and D $/ 14^{+/-}$. (C,D) Concentration response curves for norepinephrine (NE) and phenylephrine (Phe). (E) Arterial vasorelaxation in response to increasing concentration of acetylcholine (ACh). Arteries were pre-constricted with $35 \mathrm{mmol} / \mathrm{I} \mathrm{K} \mathrm{K}^{+}$Krebs buffer. (F) Arterial vasorelaxation in response to ACh after pre-treatment with the nitric oxide-synthase inhibitor L-NAME.

(G) Arterial vasorelaxation in response to increasing concentrations of the nitric oxide donor sodium-nitroprusside (SNP). Relaxation responses are comparable in D//4 ${ }^{+-}$and wild type. Wild type, $n=14 ; D / 14^{+/-}, n=13$ arteries. ${ }^{*} P<0.05$

Notch signaling positively regulates vascular maturation factors such as PDGFR-b and TGF-b (Trindade et al., 2008; Williams et al., 2006), indicating that lack of these factors in $\mathrm{Dll4}^{+/-}$mice may affect SMC coverage and function. We have indeed observed that $D l l 4^{+/-}$pial collaterals initially show a delay in SMC coverage, but this appears to be a transient defect and adult arterial SMC coverage showed no detectable difference between wild-type and Dll4 mutant littermates (data not shown). Dll4 blockade has also been shown to regulate expression of numerous chemokines, which may trigger crosstalk with inflammatory cells such as monocytes known to be required for arteriogenesis (Al Haj Zen et al., 2010; Arras et al., 1998). 

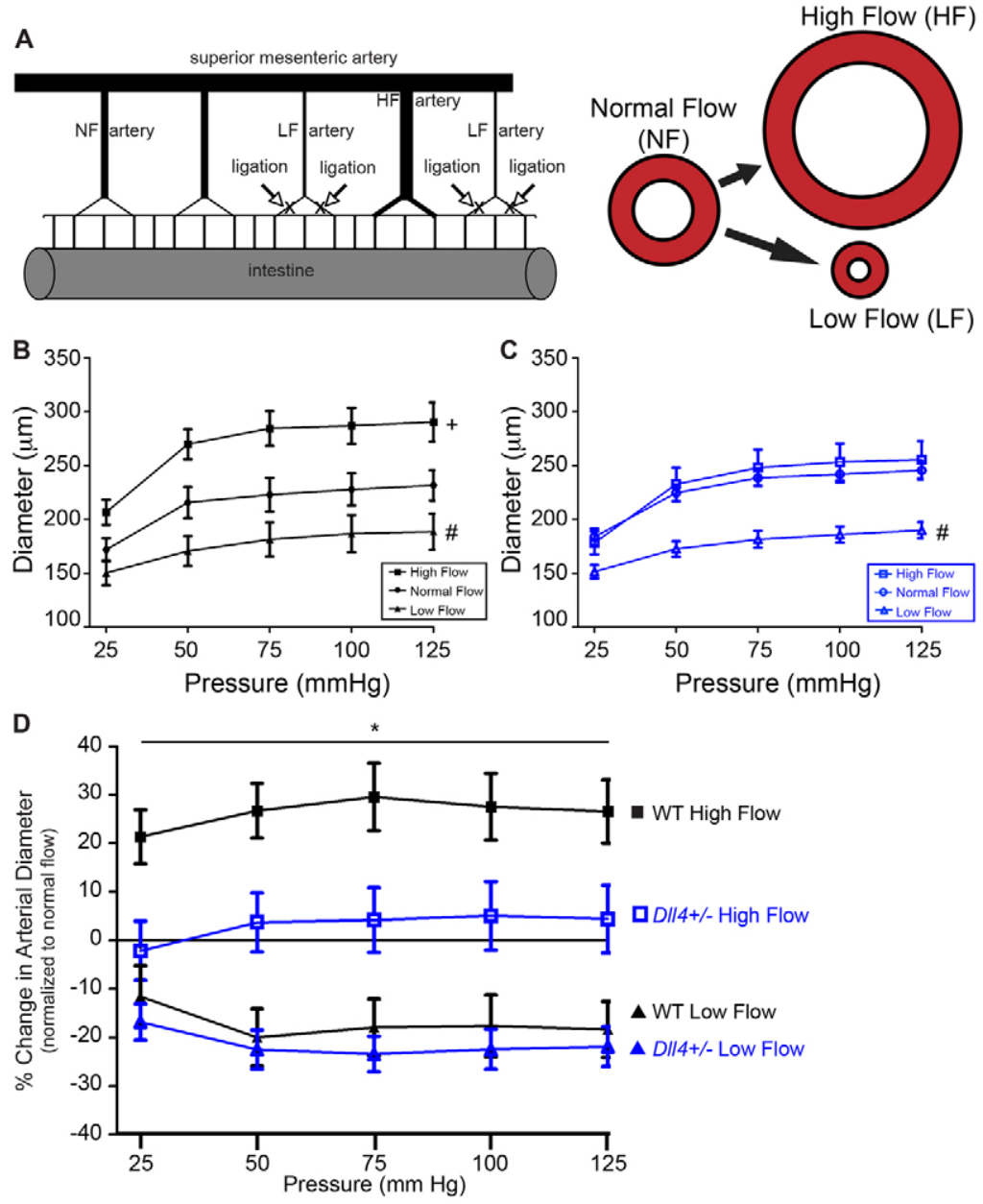

Fig. 8. Arterioles of $\mathrm{DII} 4^{+/-}$mice show reduced flow induced outward remodeling. (A) The mesenteric ligation model (left panel). Ligation of second order mesenteric resistance arteries (arrows) creates low-flow (LF artery) and high-flow arterioles (HF artery). Chronic exposure to $\mathrm{HF}$ induces outward diameter remodeling (right panel); exposure to LF induces inward remodeling (right panel), when compared with normal flow (NF). Arterioles were exposed to the indicated flow regime for 7 days, mounted in an arteriograph and passive arteriolar diameters at increasing intravascular pressures were recorded. (B) Passive diameter distension curve at increasing pressures for wild-type arterioles exposed to high flow, normal flow or low flow. (C) Passive diameter distension curve at increasing pressures for $\mathrm{D} / \mathrm{A}^{+/-}$ arterioles exposed to high flow, normal flow or low flow. (D) Remodeling responses normalized to normal flow arterioles. High flow DII ${ }^{+/-}$arterioles are structurally smaller than high flow wild type. The data are shown as mean \pm s.e.m.; wild type, $n=6$; $D \| 4^{+/-}, n=6$; ${ }^{*} P<0.05$ using ANOVA, wild-type HF (closed squares) versus DII/ ${ }^{+/-} \mathrm{HF}$ (open squares); ${ }^{~} P<0.05$ using ANOVA, NF versus LF; ${ }^{+} P<0.05$ using ANOVA, HF versus NF.
In addition to downstream regulators, upstream regulation of Dll4 expression during arteriogenesis also deserves consideration. Endothelial deletion of Hif2a has recently been shown to lead to a Dll4-dependent reduction in post-ischemic blood flow recovery, indicating that Hif $2 a$ expression controls arteriogenesis via Dll4Notch (Skuli et al., 2012). In addition to Hif2a, inducible blockade of $\mathrm{NF} \kappa \mathrm{B}$ in endothelial cells using a transgenic mouse line overexpressing I $\mathrm{KB}$ also leads to reduction in Dll4 levels, retinal hypervascularization and reduced post-ischemic blood flow recovery. Activation of Notch signaling via administration of Jag1 peptide rescues a normal vascular phenotype, indicating that NFkkB signaling, which is controlled by shear stress, regulates arterial branching morphogenesis via induction of D114-Notch signaling (Tirziu et al., 2012).

Our study may have important implications for future clinical revascularization approaches. We show that the extent of collateral network development should be delicately fine-tuned to obtain optimal flow recovery: the quality is not in numbers. We provide the proof of concept that it is possible to increase collateral number pharmacologically by postnatal treatment with a Notch inhibitor, as postnatal Notch inhibition with DAPT resulted in a sustained increase in collaterals, similar to the phenotype observed in adult $\mathrm{Dll}^{+/-}$mice. Such approaches maybe particularly important for prevention strategies. In those organs that are extremely sensitive to short-term hypoxia/ischemia insults, such as the brain, the therapeutic window is relatively small. Therefore, increasing the number of collaterals prior to stenosis may alleviate or prevent ischemic damage occurring during the early phase upon stenosis and expand the therapeutic window.

\section{Acknowledgements}

We thank Anja Zimmer for technical support.

\section{Funding}

This study was supported by grants from the Helmholtz Association and the Center for Stroke Research Berlin (FLN), Inserm, Fondation Bettencourt and the Leducq Foundation - Artemis Transatlantic network grant (to A.E., M.S., M.L.I.A.). B.C. was supported by the Fondation pour la Recherche médicale (FRM) and Fondation Leducq.

\section{Competing interests statement}

The authors declare no competing financial interests.

\section{Supplementary material}

Supplementary material available online at

http://dev.biologists.org/lookup/suppl/doi:10.1242/dev.092304/-/DC1

\section{References}

Al Haj Zen, A., Oikawa, A., Bazan-Peregrino, M., Meloni, M., Emanueli, C. and Madeddu, P. (2010). Inhibition of delta-like-4-mediated signaling impairs reparative angiogenesis after ischemia. Circ. Res. 107, 283-293.

Arras, M., Ito, W. D., Scholz, D., Winkler, B., Schaper, J. and Schaper, W. (1998). Monocyte activation in angiogenesis and collateral growth in the rabbit hindlimb. J. Clin. Invest. 101, 40-50.

Benedito, R., Roca, C., Sörensen, I., Adams, S., Gossler, A., Fruttiger, M. and Adams, R. H. (2009). The notch ligands DII4 and Jagged1 have opposing effects on angiogenesis. Cell 137, 1124-1135.

Buschmann, I., Pries, A., Styp-Rekowska, B., Hillmeister, P., Loufrani, L., Henrion, D., Shi, Y., Duelsner, A., Hoefer, I., Gatzke, N. et al. (2010). Pulsatile shear and Gja5 modulate arterial identity and remodeling events during flowdriven arteriogenesis. Development 137, 2187-2196. 
Carmeliet, P. (2000). Mechanisms of angiogenesis and arteriogenesis. Nat. Med. 6, 389-395

Cayla, C., Todiras, M., Iliescu, R., Saul, V. V., Gross, V., Pilz, B., Chai, G., Merino, V. F., Pesquero, J. B., Baltatu, O. C. et al. (2007). Mice deficient for both kinin receptors are normotensive and protected from endotoxin-induced hypotension. FASEB J. 21, 1689-1698.

Chalothorn, D. and Faber, J. E. (2010). Formation and maturation of the native cerebral collateral circulation. J. Mol. Cell. Cardiol. 49, 251-259.

Clayton, J. A., Chalothorn, D. and Faber, J. E. (2008). Vascular endothelial growth factor-A specifies formation of native collaterals and regulates collateral growth in ischemia. Circ. Res. 103, 1027-1036.

Deutsch, U., Schlaeger, T. M., Dehouck, B., Döring, A., Tauber, S., Risau, W. and Engelhardt, B. (2008). Inducible endothelial cell-specific gene expression in transgenic mouse embryos and adult mice. Exp. Cell Res. 314, 1202-1216.

Di Lorenzo, A., Fernández-Hernando, C., Cirino, G. and Sessa, W. C. (2009).

Akt1 is critical for acute inflammation and histamine-mediated vascular leakage. Proc. Natl. Acad. Sci. USA 106, 14552-14557

Duarte, A., Hirashima, M., Benedito, R., Trindade, A., Diniz, P., Bekman, E., Costa, L., Henrique, D. and Rossant, J. (2004). Dosage-sensitive requirement for mouse Dll4 in artery development. Genes Dev. 18, 2474-2478.

Eitenmüller, I., Volger, O., Kluge, A., Troidl, K., Barancik, M., Cai, W. J., Heil, M., Pipp, F., Fischer, S., Horrevoets, A. J. et al. (2006). The range of adaptation by collateral vessels after femoral artery occlusion. Circ. Res. 99 656-662.

Feil, R., Brocard, J., Mascrez, B., LeMeur, M., Metzger, D. and Chambon, P. (1996). Ligand-activated site-specific recombination in mice. Proc. Natl. Acad. Sci. USA 93, 10887-10890.

Feil, R., Wagner, J., Metzger, D. and Chambon, P. (1997). Regulation of Cre recombinase activity by mutated estrogen receptor ligand-binding domains. Biochem. Biophys. Res. Commun. 237, 752-757.

Gale, N. W., Dominguez, M. G., Noguera, I., Pan, L., Hughes, V., Valenzuela D. M., Murphy, A. J., Adams, N. C., Lin, H. C., Holash, J. et al. (2004) Haploinsufficiency of delta-like 4 ligand results in embryonic lethality due to major defects in arterial and vascular development. Proc. Natl. Acad. Sci. USA 101, 15949-15954.

Grundmann, S., Piek, J. J., Pasterkamp, G. and Hoefer, I. E. (2007) Arteriogenesis: basic mechanisms and therapeutic stimulation. Eur. J. Clin. Invest. 37, 755-766.

Hellström, M., Phng, L. K., Hofmann, J. J., Wallgard, E., Coultas, L., Lindblom, P., Alva, J., Nilsson, A. K., Karlsson, L., Gaiano, N. et al. (2007). Dll4 signalling through Notch1 regulates formation of tip cells during angiogenesis. Nature 445, 776-780

le Noble, F. A., Ruijtenbeek, K., Gommers, S., de Mey, J. G. and Blanco, C. E. (2000). Contractile and relaxing reactivity in carotid and femoral arteries of chicken embryos. Am. J. Physiol. 278, H1261-H1268.

Lobov, I. B., Cheung, E., Wudali, R., Cao, J., Halasz, G., Wei, Y., Economides, A., Lin, H. C., Papadopoulos, N., Yancopoulos, G. D. et al. (2011). The Dll4/Notch pathway controls postangiogenic blood vessel remodeling and regression by modulating vasoconstriction and blood flow. Blood 117, 67286737.

Loufrani, L., Levy, B. I. and Henrion, D. (2002). Defect in microvascular adaptation to chronic changes in blood flow in mice lacking the gene encoding for dystrophin. Circ. Res. 91, 1183-1189.

Lucitti, J. L., Mackey, J. K., Morrison, J. C., Haigh, J. J., Adams, R. H. and Faber, J. E. (2012). Formation of the collateral circulation is regulated by vascular endothelial growth factor-A and a disintegrin and metalloprotease family members 10 and 17. Circ. Res. 111, 1539-1550.

Meier, P., Gloekler, S., Zbinden, R., Beckh, S., de Marchi, S. F., Zbinden, S., Wustmann, K., Billinger, M., Vogel, R., Cook, S. et al. (2007). Beneficial effect of recruitable collaterals: a 10-year follow-up study in patients with stable coronary artery disease undergoing quantitative collateral measurements. Circulation 116, 975-983

Miquerol, L., Meysen, S., Mangoni, M., Bois, P., van Rijen, H. V., Abran, P., Jongsma, H., Nargeot, J. and Gros, D. (2004). Architectural and functiona asymmetry of the His-Purkinje system of the murine heart. Cardiovasc. Res. $\mathbf{6 3}$, 77-86

Nih, L. R., Deroide, N., Leré-Déan, C., Lerouet, D., Soustrat, M., Levy, B. I., Silvestre, J. S., Merkulova-Rainon, T., Pocard, M., Margaill, I. et al. (2012). Neuroblast survival depends on mature vascular network formation after mouse stroke: role of endothelial and smooth muscle progenitor cell coadministration. Eur. J. Neurosci. 35, 1208-1217.

Noguera-Troise, I., Daly, C., Papadopoulos, N. J., Coetzee, S., Boland, P., Gale, N. W., Lin, H. C., Yancopoulos, G. D. and Thurston, G. (2006) Blockade of Dll4 inhibits tumour growth by promoting non-productive angiogenesis. Nature 444, 1032-1037.

Phng, L. K. and Gerhardt, H. (2009). Angiogenesis: a team effort coordinated by notch. Dev. Cell 16, 196-208

Scehnet, J. S., Jiang, W., Kumar, S. R., Krasnoperov, V., Trindade, A. Benedito, R., Djokovic, D., Borges, C., Ley, E. J., Duarte, A. et al. (2007). Inhibition of Dll4-mediated signaling induces proliferation of immature vessels and results in poor tissue perfusion. Blood 109, 4753-4760.

Schaper, W. (2009). Collateral circulation: past and present. Basic Res. Cardiol. 104, 5-21.

Shutter, J. R., Scully, S., Fan, W., Richards, W. G., Kitajewski, J., Deblandre, G. A., Kintner, C. R. and Stark, K. L. (2000). DII4, a novel Notch ligand expressed in arterial endothelium. Genes Dev. 14, 1313-1318.

Skuli, N., Majmundar, A. J., Krock, B. L., Mesquita, R. C., Mathew, L. K., Quinn, Z. L., Runge, A., Liu, L., Kim, M. N., Liang, J. et al. (2012). Endothelial HIF-2a regulates murine pathological angiogenesis and revascularization processes. J. Clin. Invest. 122, 1427-1443.

Suchting, S., Freitas, C., le Noble, F., Benedito, R., Bréant, C., Duarte, A. and Eichmann, A. (2007). The Notch ligand Delta-like 4 negatively regulates endothelial tip cell formation and vessel branching. Proc. Natl. Acad. Sci. USA 104, 3225-3230.

Swift, M. R. and Weinstein, B. M. (2009). Arterial-venous specification during development. Circ. Res. 104, 576-588.

Thurston, G., Noguera-Troise, I. and Yancopoulos, G. D. (2007). The Delta paradox: DLL4 blockade leads to more tumour vessels but less tumour growth. Nat. Rev. Cancer 7, 327-331.

Tirziu, D., Jaba, I. M., Yu, P., Larrivée, B., Coon, B. G., Cristofaro, B., Zhuang, Z. W., Lanahan, A. A., Schwartz, M. A., Eichmann, A. and Simons, M. (2012). Endothelial nuclear factor- $\kappa \mathrm{B}$-dependent regulation of arteriogenesis and branching. Circulation 126, 2589-2600

Trindade, A., Kumar, S. R., Scehnet, J. S., Lopes-da-Costa, L., Becker, J., Jiang, W., Liu, R., Gill, P. S. and Duarte, A. (2008). Overexpression of deltalike 4 induces arterialization and attenuates vessel formation in developing mouse embryos. Blood 112, 1720-1729.

Williams, C. K., Li, J. L., Murga, M., Harris, A. L. and Tosato, G. (2006). Upregulation of the Notch ligand Delta-like 4 inhibits VEGF-induced endothelia cell function. Blood 107, 931-939.

Zhang, H., Prabhakar, P., Sealock, R. and Faber, J. E. (2010). Wide genetic variation in the native pial collateral circulation is a major determinant of variation in severity of stroke. J. Cereb. Blood Flow Metab. 30, 923-934.

Zhuang, Z. W., Gao, L., Murakami, M., Pearlman, J. D., Sackett, T. J., Simons, M. and de Muinck, E. D. (2006). Arteriogenesis: noninvasive quantification with multi-detector row CT angiography and three-dimensional volume rendering in rodents. Radiology 240, 698-707. 